\title{
Kock, Christian \& Lisa Villadsen (eds). 2014. Contemporary Rhetorical Citizenship (Leiden : Leiden University Press)
}

\section{Jürgen Siess}

\section{(2) OpenEdition Journals}

Electronic version

URL: http://journals.openedition.org/aad/2186

DOI: 10.4000/aad.2186

ISSN: 1565-8961

\section{Publisher}

Université de Tel-Aviv

\section{Electronic reference}

Jürgen Siess, « Kock, Christian \& Lisa Villadsen (eds). 2014. Contemporary Rhetorical Citizenship (Leiden : Leiden University Press) », Argumentation et Analyse du Discours [Online], 16 | 2016, Online since 11 April 2016, connection on 23 September 2020. URL : http://journals.openedition.org/aad/ 2186 ; DOl : https://doi.org/10.4000/aad.2186

This text was automatically generated on 23 September 2020 .

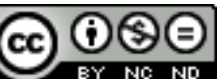

Argumentation \& analyse du discours est mis à disposition selon les termes de la licence Creative Commons Attribution - Pas d'Utilisation Commerciale - Pas de Modification 4.0 International. 


\title{
Kock, Christian \& Lisa Villadsen (eds). 2014. Contemporary Rhetorical Citizenship (Leiden : Leiden University Press)
}

\author{
Jürgen Siess
}

\section{REFERENCES}

Kock, Christian \& Lisa Villadsen (eds). 2014. Contemporary Rhetorical Citizenship (Leiden : Leiden University Press, série « Rhetoric in Society »), 272 p., ISBN : 9789087282165

1 Les éditeurs de ce collectif, chercheurs distingués de l'Université de Copenhague, renouent avec une publication remarquée de 2012, Rhetorical Citizenship and Public Deliberation. Ces deux ouvrages s'inscrivent dans un projet européen qui a à son acquis plusieurs colloques internationaux. Le projet de Kock et Villadsen se fait l'intermédiaire des recherches nord-américaines en direction de l'Europe; aussi une grande place est-elle consacrée à la rhétorique moderne et sa mise en pratique par les citoyens face aux politiciens. Les trois parties de l'ouvrage regroupent des contributions sur la rhétorique critique du point de vue du « civisme rhétorique » (I), la " mise en pratique » de ce civisme (II) et la pluridisciplinarité qu'on peut établir entre ces deux domaines (III). Le concept de rhetorical citizenship implique que les publics sont en constante évolution et que les citoyen/ne/s y participent en exerçant leur jugement pratique. La démocratie y est vue bien plus comme une « ligne directive qui façonne les interactions » que comme un cadre institutionnel (9-10). On pourrait formuler l'enjeu de ce volume sous la forme de deux questions. Comment former des acteurs qui soient compétents et qui réfléchissent, qui puissent contribuer à la constitution d'un «système de délibération efficace " (15) ? Comment préparer le grand nombre à trouver accès à l'espace de délibération largement influencé par les médias, qui peuvent manipuler le public $(16,24)$ ? On note qu'en affirmant We as citizens have the 
right to expect [...] - les directeurs du volume eux-mêmes se situent explicitement non seulement dans le champ de la recherche universitaire mais aussi dans celui de la politique : ils plaident en effet pour la mise en pratique d'une rhétorique engagée qui vise à dépasser le clivage entre politiciens et public.

2 Le volume s'ouvre par un essai substantiel dû à un des grands spécialistes nordaméricains de rhétorique, David Zarefsky : Is Rhetorical Criticism Subversive of Democracy? L'auteur observe, dans le domaine de la "critique rhétorique», une tendance à une préférence critique pour le négatif (45) qui ne laisse guère de place à une appréciation des possibilités que recèle la rhétorique actuelle. Dans sa forme extrême, ce scepticisme risque de saper la rhétorique qui se sait redevable de la démocratie délibérative. La forme même de ce texte mérite l'attention: Zarefsky procède tout au long de son article par un jeu d'assertions et d'interrogations mettant à l'épreuve chacune de ces assertions (qui sont souvent des thèses reprises à d'autres auteurs). On assiste ainsi à une sorte de dialogue du critique avec lui-même qui est cependant destiné aux lecteurs/lectrices, invitées à réfléchir avec le critique dans le but d'appréhender la rhétorique d'une façon qui permette d'évaluer les discours situés dans l'espace public, mais aussi de sonder les opportunités que peuvent avoir les citoyen/ne/s de participer au débat politique: We need to find and produce criticism of rhetorical moments when ideology is present and yet not deterministic (45).

3 Les deux chapitres qui suivent soulèvent la question de la crédibilité qui est cruciale pour le rapport que l'auditoire peut entretenir avec l'instance qui a le pouvoir de décision. Paula Olmos analyse une controverse aux enjeux sociaux et éthiques, On Rhetorical Ethos and Personal Deeds. En 2011, le professeur Francisco Rico avait déclenché une polémique avec un article dans le quotidien espagnol El Pais, "Théorie et pratique de la loi contre les fumeurs ». Après avoir défendu les fumeurs, il affirmait dans un «postscriptum » que de sa vie il n'avait fumé une seule cigarette. Ce P. S. lui valut de multiples réactions indignées. Olmos entend montrer que les opposants privilégient la question de la validité de l'argument et de la sincérité du proposant au détriment du problème de fond, de l'enjeu médical, social et économique. A ses yeux, les adversaires de Rico confondent le chercheur connu qui intervient dans le débat public avec la personne privée. C'est l'enjeu situé dans le contexte du débat citoyen qui préoccupe Rico, et Olmos met en valeur l'éthique dont il peut se réclamer, éthique centrée sur l'intérêt public, conçue comme prévalant sur l'éthique à laquelle s'arrêtent ses détracteurs, qui s'attache quant à elle aux vices et aux vertus de la personne privée. La question du rapport gouvernés-gouvernants croise ici celle du double statut de la personne - le statut de l'universitaire connu et celui de l'homme privé.

Charlotte Jorgensen consacre un article très pointu aux promesses lancées par les hommes et les femmes politiques dans le débat public au Danemark. Elle distingue deux acceptions de sens de promise/Versprechen, un sens fort - engagement qui est jaugé par le résultat - et un sens faible - but qui peut être infléchi en raison de circonstances qui ont changé. Le sens fort de broken promise est volontiers mis en avant par des concurrents politiques ou des journalistes qui se dressent contre celui/celle qui a pris tel ou tel engagement en période électorale. L'auteure plaide pour que l'analyste des débats publics donne la priorité à l'acception faible : la question serait de savoir quelle est, dans l'engagement pris par rapport au public, la marge acceptable qui maintient la crédibilité et permet aux électeurs de participer au débat en tant que citoyens "rhétoriques", engagés, reconnus comme partenaires à part entière. On peut 
regretter cependant que l'auteure donne parfois plus d'importance à la fairness (dont on aurait voulu une définition plus précise) qu'a la crédibilité, élément crucial - comme elle l'a bien vu - dans les rapports entre politiciens et électeurs.

Avec Invoking American Civil Religion in FDR's Rhetoric of Tax Equity and Citizenship, Nathalie Kuroiwa-Lewis est au coeur du sujet. Elle montre que l'argument de l'impôt progressif et l'accent mis sur les impôts conçus comme «contrat social» (terme davantage redevable au christianisme qu'à Rousseau) créent un cadre rhétorique qui permet de forger un « argument moralement et politiquement viable dans l'espace civique » pour engager les citoyens en faveur de la justice sociale. Franklin D. Roosevelt recourt à un exemple historique : les Britanniques de 1776 apparaissent comme le modèle des riches qui refusent de payer plus d'impôts, et les colons qui se dressent contre la monarchie britannique au nom d'une valeur supérieure (la liberté) comme le modèle du président qui entend guider la nation au nom d'une autre valeur supérieure (la justice sociale). Anne Ulrich traite de The West Wing en faisant l'hypothèse que cette série télévisuelle constitue un forum alternatif pour «la délibération publique et la formation au civisme ». Bien qu'elle mette en relief la double perspective (avant-scène et coulisses) sur laquelle le scénariste Aaron Sorkin joue dans sa mise en scène de la politique du White House, l'auteure risque de confondre la dimension de l'action politique à Washington et celle de la fictionnalisation de cette action. Si la double perspective est considérée comme le moyen de mettre à distance la politique réelle et de solliciter un public de citoyens avertis, on ne voit pas en quoi cela permettrait aux téléspectateurs de participer au processus de " "faire" de la politique» (136). On se demande si cela ne supposerait pas une interaction des producteurs de la série et de l'auditoire, comme Bertolt Brecht l'avait proposé, dès 1932, pour la radio comme «appareil de communication ». Ulrich présuppose en effet, de la part du public, la possibilité à la fois de s'identifier aux personnages et aux situations politiques sur l'écran et, grâce à la «double perspective » de la production, de participer 'activement' au débat politique. Les deux semblent s'exclure, puisque une telle participation implique la réflexion critique et l'accès à l'«appareil de communication" qui sont contraires à l'identification. Pour citer Sorkin lui-même: How do you establish the vocabulary with the audience? How do I set up the rules with viewers that from time to time, this is going to happen and you shouldn't be upset by it?(http://www.westwingepguide.com/s2/Episodes/) On voit qu'il ne conçoit pas ici les spectateurs comme participants actifs : pour cela, ils devraient être mis en position d'intervenants dans la production de la série au même titre que Sorkin et son équipe.

La Deuxième Partie s'ouvre sur Rhetorical Citizenship in Public Meetings. Karen Tracy traite ici du rapport entre expression de la base et démocratie représentative, dans le cas d'enjeux politiques controversés; son corpus est constitué par des hearings où est débattue la question des mariages d'homosexuels. Elle fait l'hypothèse que ces auditions publiques permettent d'accentuer le désaccord, de clarifier les enjeux et de mieux comprendre les différences d'opinion, mais qu'elles sont aussi un élément constitutif de la démocratie pluraliste, par exemple quand arguments religieux et laïques sont confrontés les uns aux autres. Les témoignages dus à des citoyens fonctionnent à la fois comme plaidoirie et comme expression passionnée d'une opinion. Aussi Tracy suggère-t-elle que ce type d'expression devrait faire partie de toute notion de civisme rhétorique (161). Elle considère ces prises de positions comme un apport décisif à une démocratie vivante. Les références à la religion, dès lors qu'elle diffère du dogme ecclésiastique et peut être opposée à celui-ci, servent ici d'arguments pour ou 
contre une revendication ou un projet de loi. - On rapprochera ce chapitre de Argumentative Literacy and Rhetorical Citizenship par Fotini Egglezou qui soulève une question très intéressante: Comment rendre des élèves sensibles à l'argumentation face à un problème éthique et social et comment les préparer à s'engager pour trouver une solution? Notons le choix de sa rhétorique d'appui : s'inspirant d'Isocrate, l'auteur se distingue des aristotéliciens qui tiennent le haut du pavé dans ce volume. La sagesse pratique prônée par Isocrate est mobilisée pour guider les élèves dans une "enquête sur le bien » qu'ils doivent mener collectivement (184). L'étude empirique réalisée avec 24 collégiens part du problème des aliments génétiquement modifiés. L'auteur rapporte qu'ils ont pu découvrir les marqueurs argumentatifs susceptibles de faciliter «la représentation de leurs identités et la négociation de leurs points de vue » (186), et qu'ils ont pu apprendre des vertus civiques comme l'empathie et la tolérance. Ce projet à l'intérieur de l'institution scolaire comportait plusieurs volets, pratiques orales et écrites d'information et de revendication, pratiques supposées aboutir à un jugement et à des conséquences, tant dans leur comportement en privé et en public que dans le domaine économique (lettre rédigée à l'intention de plusieurs entreprises de production laitière). Egglezou conclut que les élèves sont parvenus à "intérioriser ", face à une situation donnée, une argumentation qui intègre une attitude critique d'une part, l'évaluation des alternatives suivie d'un choix très pratique, d'autre part. On peut considérer comme complémentaire de cet article celui de Rebecca A. Kuehl (Partie III) qui propose d'intégrer la rhétorique citoyenne dans une pratique plus étendue, transculturelle, un « civisme mondial » (global citizenship).

Carolyne Lee et Judy Burnside-Lawry s'intéressent à l'interrelation de la voix, du récit et de l'écoute comme structure communicationnelle du civisme rhétorique, et analysent des discussions sur des enjeux sociaux telles qu'elles sont menées dans des petits groupes. Elles considèrent cette délibération en cercle restreint comme le tremplin de débats qui se déploient dans une sphère publique plus étendue. Aux yeux des auteures, la rhétorique permet de donner voix à une idée, de s'engager dans le discours public, dans l'action communicative (168-69). Elles conçoivent le civisme rhétorique comme une dynamique dans laquelle l'articulation d'une position propre appelle l'écoute et la prise en compte d'autres positions, et la réponse appropriée à celles-ci. Les auteures centrent leur propos sur la fonction du récit (narrative/story), en présupposant avec W. Fisher ( «homo narrans») que les récits sont un instrument majeur de toute tentative de persuasion (171). Le récit sert la " production de sens » qui est supposée amener un changement et obtenir un gain politique. L'article établit un lien entre "micro-» et "macro-public», et présente la situation actuelle comme défavorable à la démocratie : l'importance de la «voix» du citoyen serait diminuée au profit d'une gouvernance qui s'appuie sur la prédominance du marché. Le but des auteures consiste donc à apporter des éléments permettant de contrecarrer une situation qui fait fi du civisme rhétorique. Ici, la voix prend toute son importance : elle participe à la fois de la réflexion et d'un échange qui intègre des récits mais constitue aussi l'aptitude de chacun à la "coopération sociale» (173). L'étude empirique de conversations entre étudiants d'université confirme la fonction de la "production mutuelle de sens » et la place du récit personnel dans la relation qui s'établit entre les différents points de vue (on aurait cependant souhaité voir un corpus plus conséquent). Lee et Burnside supposent qu'on peut très bien transposer leur modèle basé sur un espace public restreint à l'espace public élargi, par exemple en analysant les points de vue qui s'expriment dans une confrontation entre citoyens et organisations (politiques 
ou économiques) au sujet d'un projet qui implique la collectivité (178). On attend la suite. - Il faut mentionner ici aussi l'article de Deneire, Eelbode et Lauwers qui traite de la persuasion en proposant d'intégrer rhétorique et Game Theory, article intéressant sans doute mais qui dépasse mes compétences.

On change de cadre méthodologique et de culture avec Mari Lee Mifsud qui se propose de déceler les éléments du civisme rhétorique chez les Anciens : Aristote, Homère et Aristophane. Elle conçoit le krites d'Aristote comme un citoyen qui doit juger les affaires courantes de la vie civile (226). Elle note qu'Aristote cite l'éloquence et la capacité d'action comme don (given good), sens qu'on trouve déjà dans l'Iliade, mais aussi dans Lysistrata où (aux yeux de Mifsud) le personnage principal agit face aux magistrats comme un "simple juge qui n'a pas besoin de qualifications ni de raisonnements spécieux " en demandant de faire une offre civique, solidaire, qui permettra d'éviter la guerre avec l'ancien allié $(229,232)$. Lysistrata peut ainsi apparaître comme le modèle de la citoyenne responsable, s'engageant pour le bien public. - On lira comme une suite de ce chapitre celui de Raymie E. McKerrow, The Rhetorical Citizen: Enacting Agency. On le verra plus précisément comme une adaptation du problème discuté par Mifsud au civisme rhétorique à l'époque moderne. A présent, c'est de se faire reconnaître qu'il s'agit, pour sa capacité de parler ou d'écrire en public qui est une capacité d'action, l'habileté du locuteur-scripteur à répondre à des situations changeantes et à "négocier les positionnements sociaux" (240). Au centre de la scène, McKerrow place les exclus, en posant la question de savoir comment ils peuvent subvertir l'exclusion qui les frappe. Comment faire entendre leur voix et accéder ainsi au statut de citoyen "rhétorique », actif? En assumant pleinement le statut de part of those that have no part, car plus le sujet explicite le modèle qui le détermine, plus il se donne le pouvoir d'agir (243). Des manifestants considérés comme des illégaux peuvent endosser le rôle de citoyens reconnus comme tels grâce à leurs formes d'engagement, et " prouver leur citoyenneté en accomplissant au quotidien des actes rhétoriques ». Comme exemple probant l'auteure cite le cas des femmes antiesclavagistes du $19 \mathrm{e}$ siècle et soutient que l'acte même de formuler des pétitions devient ici une forme d'engagement: elles furent citoyennes "grâce aux diverses procédures auxquelles elles eurent recours en essayant de se faire entendre " (245). Mentionnons ici deux autres articles qui évoquent des "actes symboliques» de protestation, "On Trees" par Kati Hannken-Illjes et "Yarn Bombing" par Maureen Daly Gogging.

9 La Troisième Partie, Crossing Borders, s'ouvre sur une mise au point théorique de Peter Dahlgren qui plaide pour une conception susceptible de croiser la rhétorique avec la Critical Discourse Analysis (Fairclough et Wodak) et la Théorie du Discours (Laclau et Mouffe). Sa réflexion part de son propre domaine d'expertise, les sciences de la communication centrées sur l'étude du Web. L'hypothèse est que le Web est devenu l'espace public dominant où, en dépit de tares et risques divers, le civisme rhétorique peut se déployer (257). On remarque que Dahlgren émet la réserve que se mettre en ligne ne fait pas des citoyens actifs de gens qui ne sont pas engagés ailleurs; il note les inconvénients et les dangers que comporte le nouveau média : prédominance d'intérêts commerciaux, cercles stériles de gens partageant la même opinion, consommation de données sans engagement, motifs et modes anti-démocratiques, cacophonie et manque de formatage. Il conçoit néanmoins le Web comme une ressource vitale pour la participation citoyenne (261). Il soutient que, dans la recherche en communication, l'accent se déplace de l'allocutaire (l'auditoire) vers le scripteur considéré non plus 
comme manipulable mais comme un agent actif produisant du sens, capable de parvenir à l'indépendance, voire à une approche critique (263). Résumant les apports de la CDA et de la Discourse Theory, l'auteur plaide pour leur insertion dans la rhétorique des médias, suggérant de prêter une attention particulière aux rapports de pouvoir qui permettent la participation citoyenne autant qu'ils la restreignent (269). - Ajoutons ici que l'article "People Power" in Philippine Presidential Rhetoric (par Gene Segarra Navera) traite de la manipulation, dans un autre genre et davantage en termes de linguistique : il montre comment certains leaders affirmant qu'ils veulent passer à la démocratie se servent du terme " pouvoir du peuple » dans une acception ambiguë ou populiste.

Hilde van Belle met en valeur un écrivain immigré qui s'engage dans le débat public en se faisant le défenseur de la démocratie. Le Marocain-Néerlandais Hafid Bouazza polémique contre le type de débat qui évite le conflit des idées et soutient que la political correctness est vouée à échec face à la violence du discours populiste, qu'il soit de tendance fasciste, fondamentaliste ou d'extrême-droite. Face à l'Islam extrémiste dans lequel convergent idéologie totalitaire et agression machiste, il rappelle aux Occidentaux leurs propres combats du passé avec les institutions religieuses et la répression. Van Belle conçoit la polarisation entre fanatisme et liberté de la parole que pointe Bouazza comme renvoyant à la prise de conscience d'un profond conflit entre pensée religieuse et politique démocratique (284).

11 Catherine Chaput, dans Rhetorical Citizenship beyond the Frontiers of Capitalism, développe une critique qui sort avantageusement du cadre que la plupart des contributeurs respectent (parfois trop, peut-être). Elle déconstruit les notions de démocratie et de rhétorique selon la doxa, notions dont elle retrace (à la suite de G. Arrighi) l'interrelation étroite à travers l'histoire depuis la Renaissance. La "démocratie " dominante est celle qui a fait un pacte avec le capitalisme, le point culminant se situant au $18^{\mathrm{e}}$ siècle, en Grande-Bretagne : s'est construit depuis lors le mythe d'une association tripartite. Ce mythe de l'« individualisme capitaliste » associée à la démocratie libérale et à la rhétorique moderne doit toujours être réaffirmé (311), la rhétorique est supposée le propager (la chute du communisme d'Etat en 1989 a fourni un prétexte à cette propagation) - et si civisme il y a, c'est sans faire une place au discours de la démocratie active, critique, participative. Chaput fonde sa conception critique de la démocratie sur un argument socio-économique, en s'appuyant sur le film Marx Reloaded de Jason Barker. Le réalisateur propose une vision alternative qui s'inspire du collectivisme comme forme d'organisation à orientation sociale. Marx Reloaded adapte cependant les deux visions, capitaliste et communiste, en plaidant pour une critique de la démocratie libérale liée au capitalisme et une réinterprétation de la valeur d'usage comme valeur sociale, collective en faveur des producteurs, au détriment des propriétaires. Barker en induit la possibilité de replacer la démocratie dans le contexte communiste (communist horizon) : la prémisse est ici que le « commun " (la collectivité) crée des produits mais aussi des pratiques sociales, des modes de vie, des langages. Aussi s'agit-il, aux yeux de Chaput, de renouer avec des formes de production collective et coopérative et de développer un sens nouveau de la citoyenneté, celui d'un civisme actif susceptible de constituer un nouveau public $(317,319)$.

La place centrale qui, dans ce volume, est conférée à la question des fonctions citoyennes de la rhétorique devrait tout particulièrement retenir l'attention des chercheurs francophones, qui se penchent beaucoup moins que leurs homologues étrangers sur l'apport de la rhétorique à la vie en démocratie. Par l'importance de ses 
enjeux, cet ouvrage mérite par ailleurs une large audience au-delà des spécialistes en argumentation, en études de la communication et en sciences politiques.

\section{AUTHORS}

\section{JÜRGEN SIESS}

Université de Caen, ADARR 